\title{
HYDRODYNAMIC MODELING OF MINERAL WOOL FIBER SUSPENSIONS IN A TWO-DIMENSIONAL FLOW
}

Gregory M. Cartland Glover, Alexander Grahn, Eckhard Krepper, Frank-Peter Weiss
Sören Alt, Rainer Hampel, Wolfgang Kästner, Alexander Kratzsch, Frank Zacharias

Hochschule Zittau-Görlitz Institut für Prozeßtechnik, Prozeßautomatisierung und Meßtechnik
Forschungszentrum Dresden-Rossendorf Institut für Sicherheitsforschung 


\section{HYDRODYNAMIC MODELING OF MINERAL WOOL FIBER SUSPENSIONS IN A TWO-DIMENSIONAL FLOW}

- Motivation

- Problem

- Experiment

- Numerical Model

- Results

- Conclusions

- Future Work 


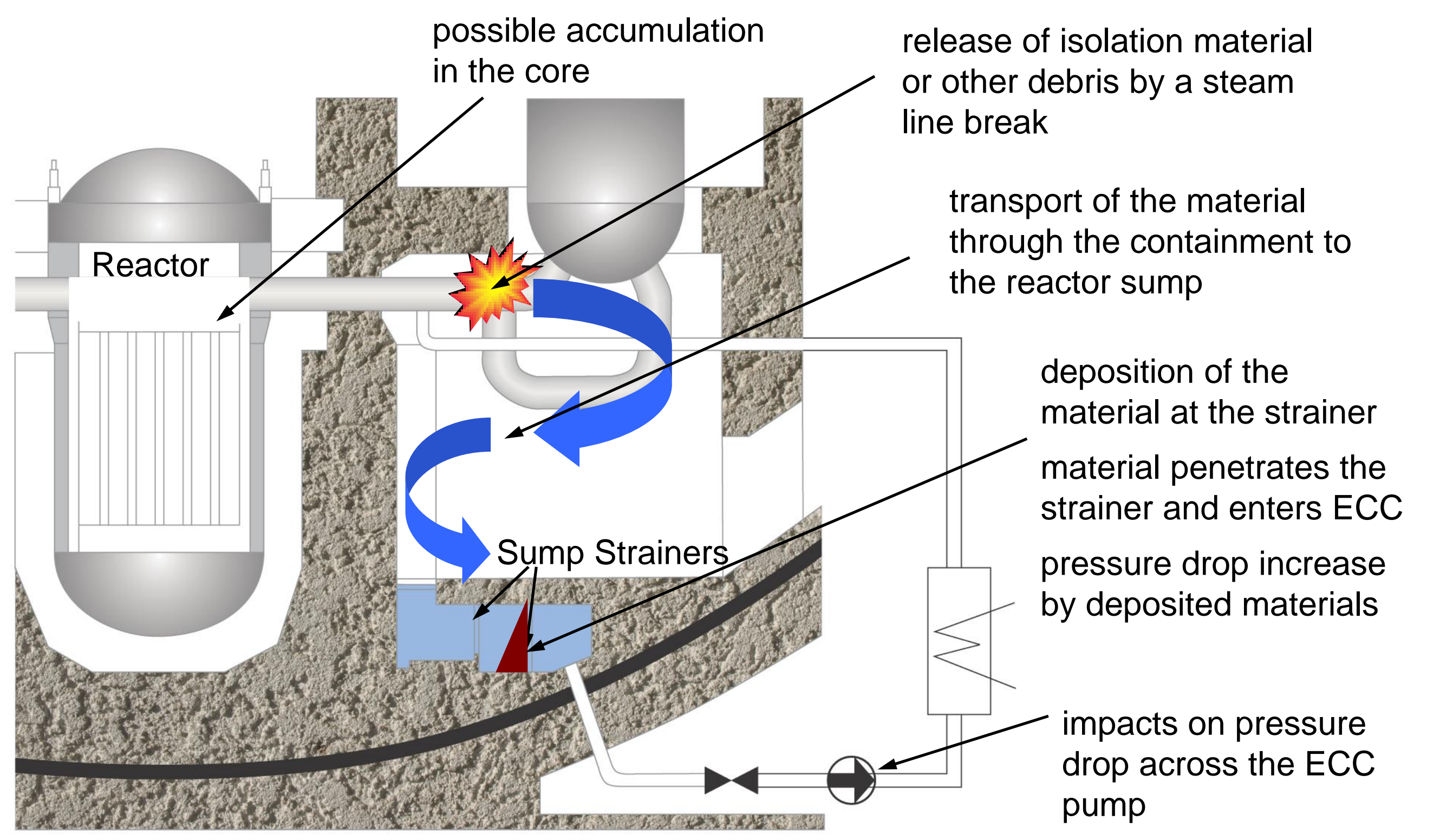




\section{Research project funded by the German Federal Ministry of Economy and Technology}

Project No. 150 1270, 150 1307, 150 1360, 1501363

- General aim:

- single effect and combined effect tests on fibrous insulation materials

- model development and validation

- application of CFD models

- later integration into containment code systems

- project is not part of the oversight process

- it is not the immediate goal to deliver any safety proof 


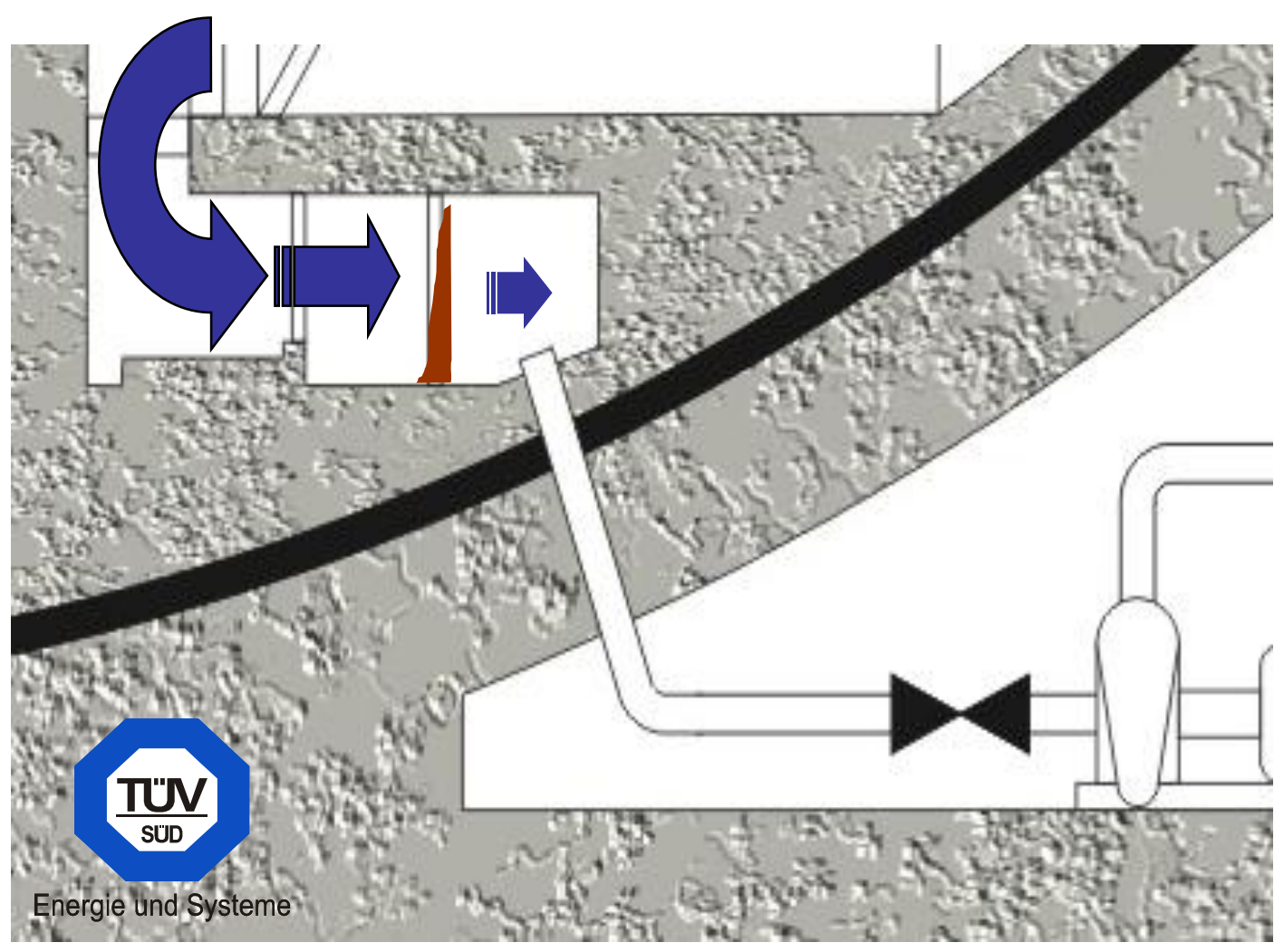

insulation materials are transported to the containment sump

sedimentation and resuspension affect material through the sump

descending water jets and turbulence agitate the liquid and materials in the sump

materials may accumulate on or penetrate the strainer 


\section{Development and validation of numerical models for insulation material transport in the sump}

- Aim:

- single effect tests of material transport phenomena

- CFD model development and validation

- sedimentation and resuspension are key phenomena

- Experiment

- racetrack type channel studied phenomena under different horizontal flow conditions

- Numerical Model

- Eulerian-Eulerian models of whole and channel straight sections show accumulation at the lower boundary 


\section{Racetrack Channel}

- $\sim 0.4 \mathrm{~kg}$ steam-blasted MDK mineral wool fibers

- Recirculated at velocities of $0.1,0.2$ and $0.5 \mathrm{~m} \mathrm{~s}^{-1}$

- Lasers at 0.1, 0.2, 0.3 and $0.4 \mathrm{~m}$ measure turbidity $\sim 2 \mathrm{~m}$ upstream of the impellers

- Images taken of fiber accumulation at the base

- No baffles

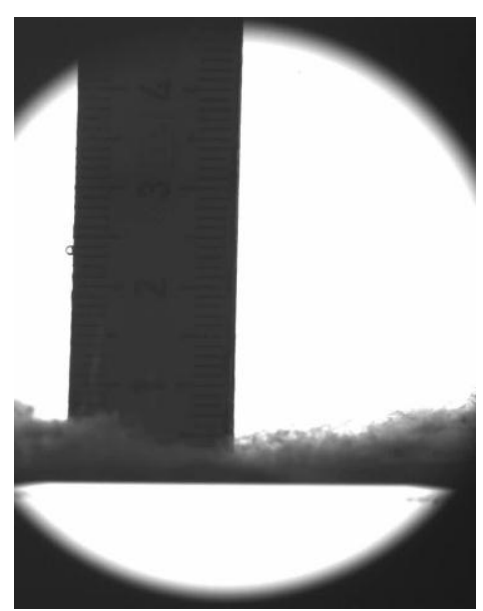

Calibration

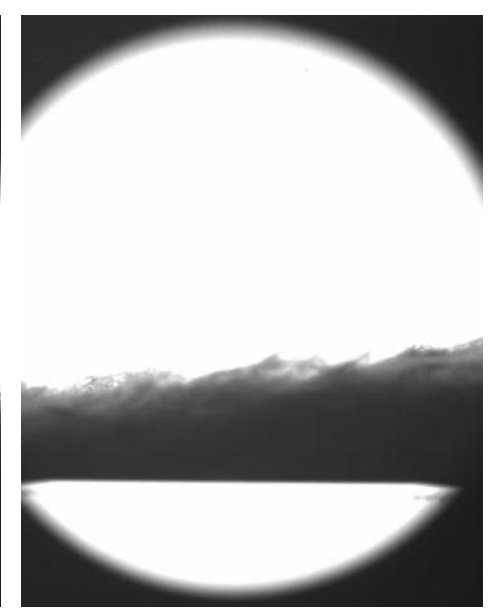

$0.1 \mathrm{~m} \mathrm{~s}^{-1}$

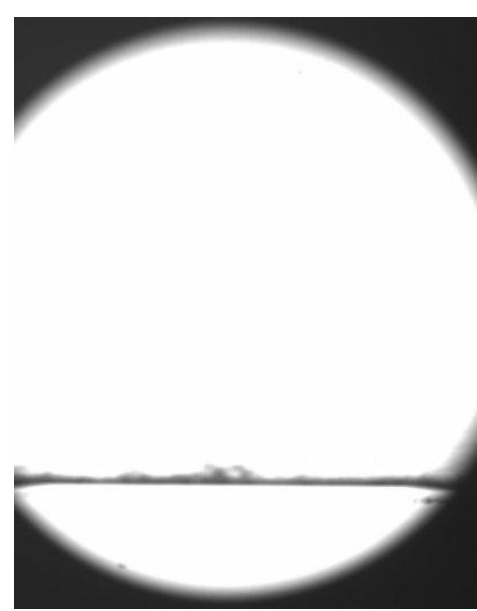

$0.2 \mathrm{~m} \mathrm{~s}^{-1}$ 


\section{Racetrack Channel: Relative Turbidity}
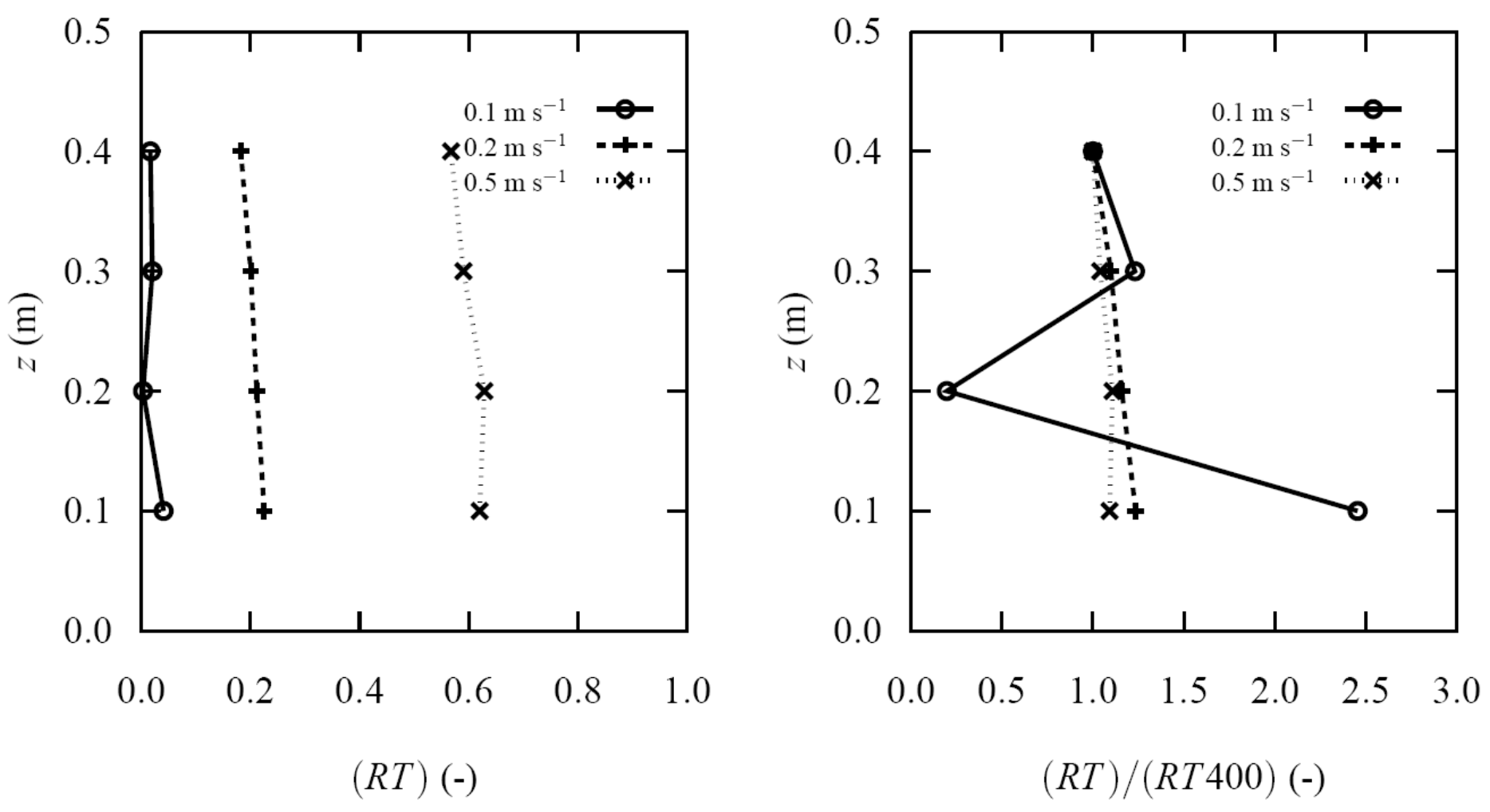

Averaged profiles of last 10 minutes of each run
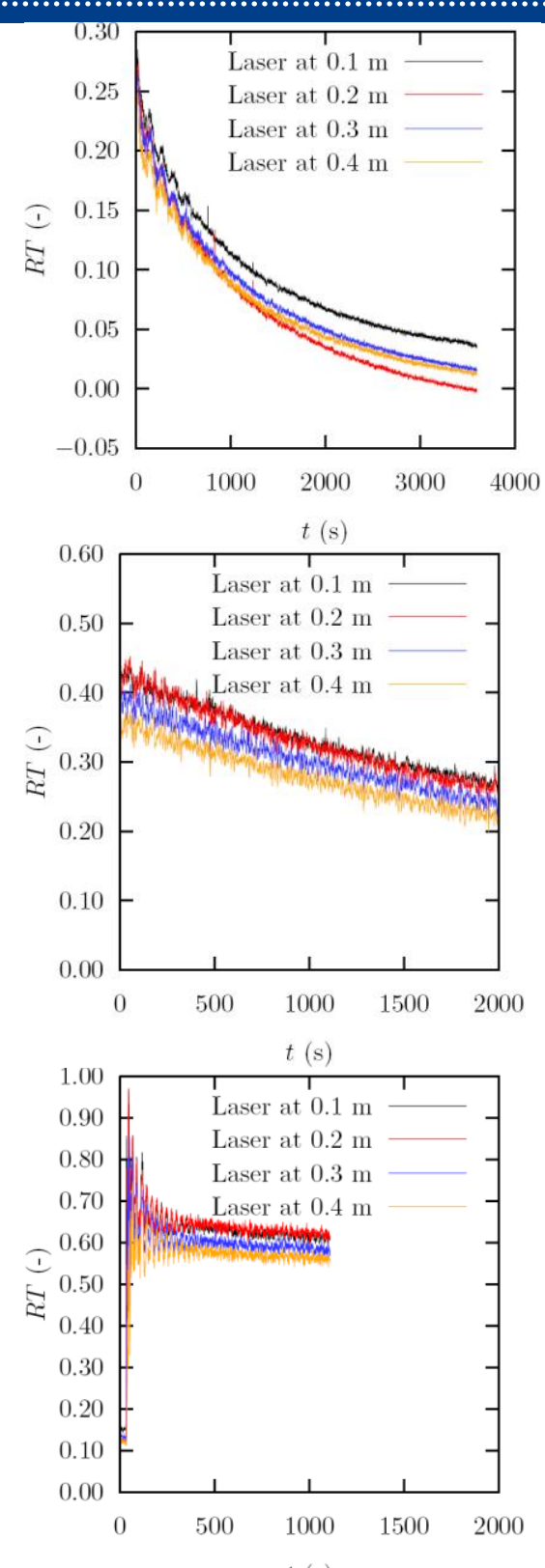


\section{Channel section}

- $3 *{ }^{*} 0.1 \mathrm{~m}^{3}$ section upstream of the impellers

- Near uniform velocity conditions

- Eulerian-Eulerian multifluid model

- SST turbulence model with automatic functions

- $\mathrm{y}+\sim 100$

- Two Drag Force Correlations

- Schiller-Naumann, $C_{D, S N}$

- Wall modified Schiller-Naumann $C_{D, S N W}$

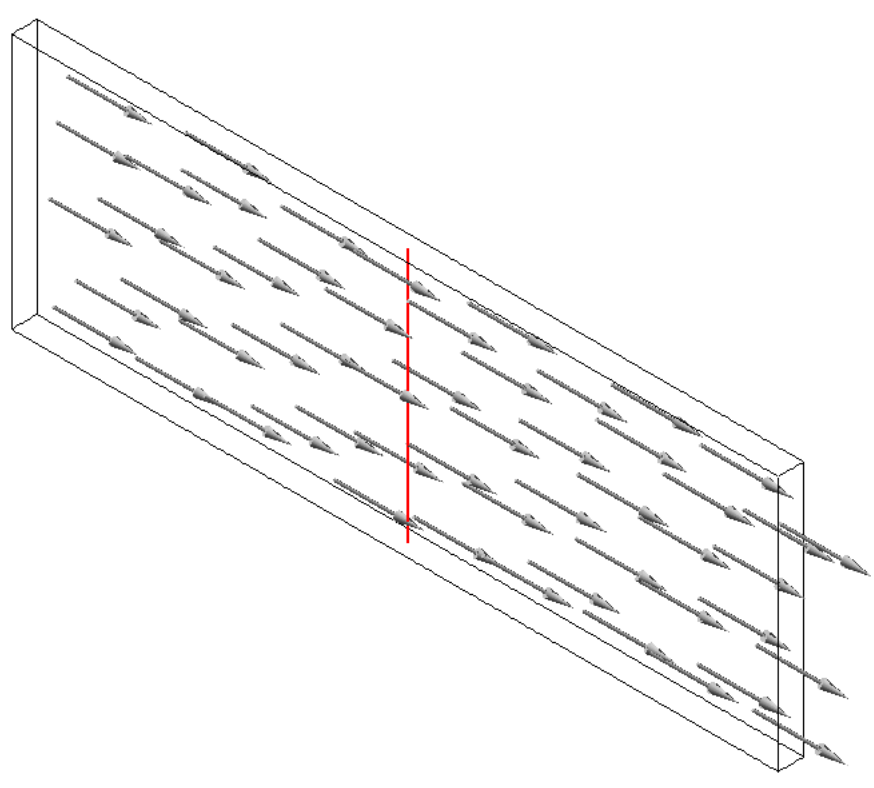

- Favre averaged turbulence dispersion force

- Water - Virtual spherical particle

- $\mathrm{d}_{\mathrm{p}}=5 \mathrm{~mm}$

- Relative viscosity of dispersed phase based on Batchelor (1977) J. Fluid Mechanics 83, 97

$$
\mu_{r}=1+2.5 r_{p}+7.6 r_{p}^{2}
$$




\section{Channel section}

\begin{tabular}{llll}
\hline Phase & $\rho_{\mathrm{p}}$ & $\mathrm{u}_{\mathrm{tp}}$ & $\zeta_{\mathrm{p}}$ \\
\hline $\mathrm{L}$ & 1002 & $\sim 0.02$ & 0.01664 \\
$\mathrm{H}$ & 1027 & $\sim 0.05$ & 0.00277 \\
\hline
\end{tabular}

$$
u_{t p}=\sqrt{\frac{4}{3} g \frac{\rho_{p}-\rho_{c}}{\rho_{c}} d_{p} \frac{1}{C_{D}}}
$$$$
\zeta_{p}=\frac{\rho_{p}-\rho_{c}}{\rho_{f}-\rho_{c}}
$$$$
r_{p}=\left\{\begin{array}{cc}
r_{p, \max } & z \leq 0.02 m \\
0 & z>0.02 m
\end{array}\right.
$$

\begin{tabular}{llllll}
\hline Condition & $\begin{array}{l}\text { Inlet } \\
\text { Velocity }\end{array}$ & $10^{3 *} \mathrm{Re}$ & $\mathrm{r}_{\mathrm{p}}$ & \multicolumn{2}{c}{ Fiber Transport } \\
\cline { 3 - 5 } & $\left(\mathrm{m} \mathrm{s}^{-1}\right)$ & & $\mathrm{H}$ & $\mathrm{L}$ & \\
\hline $\operatorname{Re}_{\mathrm{A}}$ & 0.5 & 101.8 & 0.0146 & 0.0069 & Suspended \\
$\operatorname{Re}_{\mathrm{B}}$ & 0.2 & 40.7 & 0.0146 & 0.0069 & Mixed \\
$\operatorname{Re}_{\mathrm{C}}$ & 0.1 & 20.4 & 0.4167 & 0.1942 & Sedimented \\
\hline
\end{tabular}




\section{One dispersed phase:}

Interfacial Force Coefficients: $\mathrm{C}_{\mathrm{TD}}=1 ; \mathrm{C}_{\mathrm{D}, \mathrm{SN}}$;

Three inlet velocities: $\mathrm{Re}_{\mathrm{A}} \approx 10^{5} ; \mathrm{Re}_{\mathrm{B}} \approx 4^{*} 10^{4} ; \mathrm{Re}_{\mathrm{C}} \approx 2^{*} 10^{4}$;

Comparison of dispersed phase densities: $\rho_{\mathrm{pL}}=1002 \mathrm{~kg} \mathrm{~m}^{-3} ; \rho_{\mathrm{pH}}=1027 \mathrm{~kg} \mathrm{~m}^{-3}$;
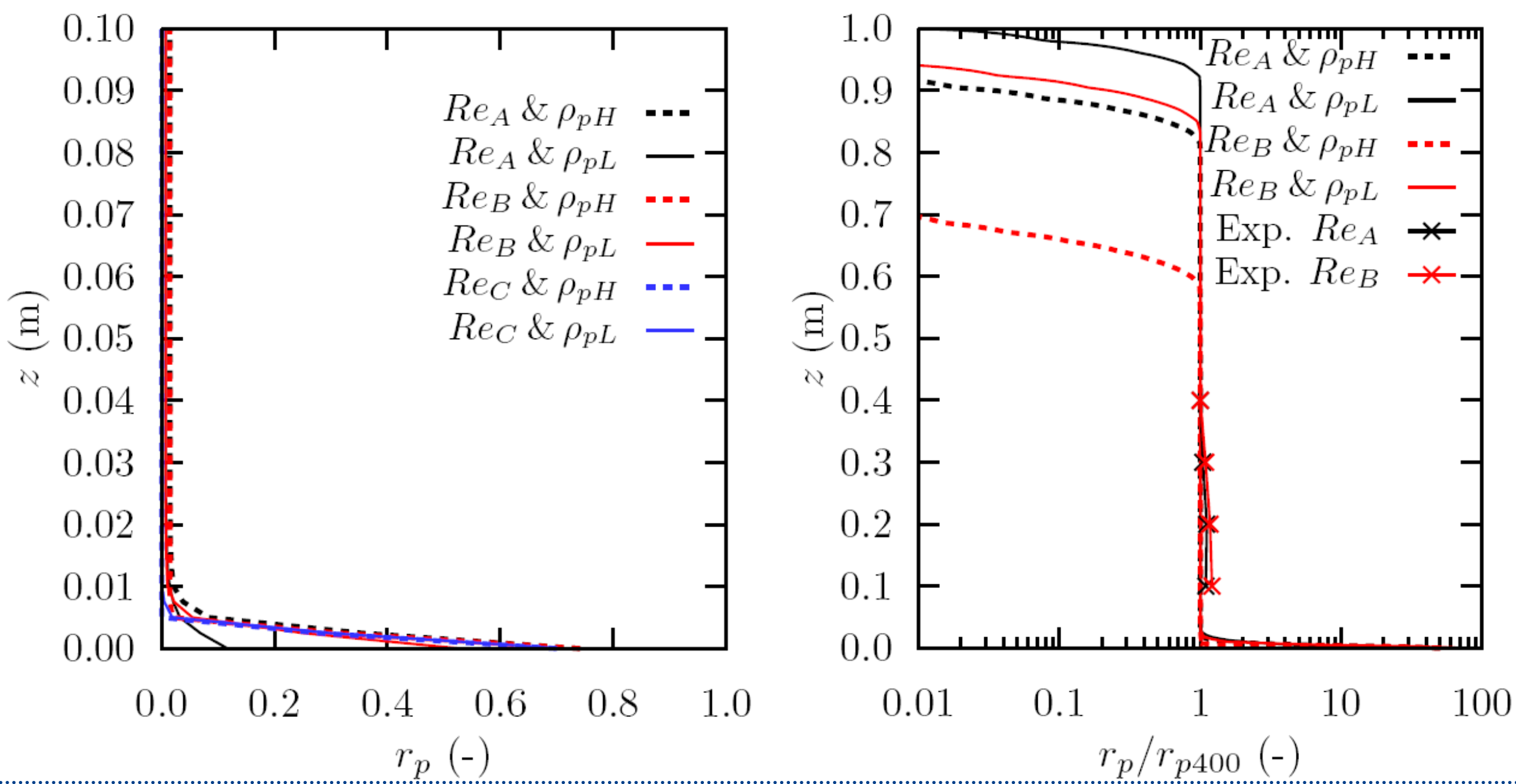


\section{One dispersed phase:}

Interfacial Force Coefficients: $\mathrm{C}_{\mathrm{TD}}=1 ; \mathrm{C}_{\mathrm{D}, \mathrm{SNW}}$;

Three inlet velocities: $\mathrm{Re}_{\mathrm{A}} \approx 10^{5} ; \mathrm{Re}_{\mathrm{B}} \approx 4^{*} 10^{4} ; \mathrm{Re}_{\mathrm{C}} \approx 2^{*} 10^{4}$;

Comparison of dispersed phase densities: $\rho_{\mathrm{pL}}=1002 \mathrm{~kg} \mathrm{~m}^{-3} ; \rho_{\mathrm{pH}}=1027 \mathrm{~kg} \mathrm{~m}^{-3}$;
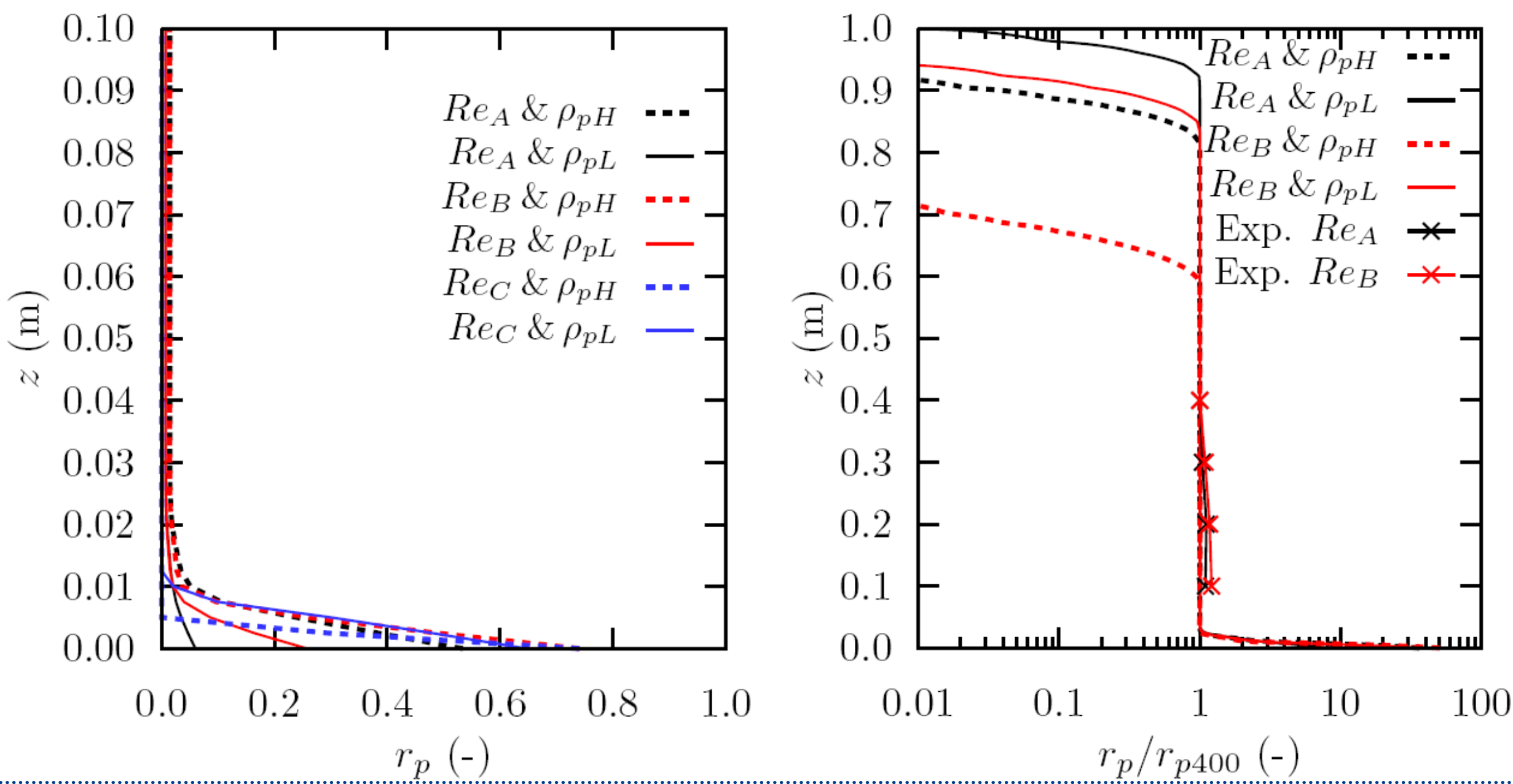


\section{One dispersed phase:}

Interfacial Force Coefficients: $\mathrm{C}_{\mathrm{TD}}=100 ; \mathrm{C}_{\mathrm{D}, \mathrm{SN}}$;

Three inlet velocities: $\operatorname{Re}_{\mathrm{A}} \approx 10^{5} ; \mathrm{Re}_{\mathrm{B}} \approx 4 * 10^{4} ; \mathrm{Re}_{\mathrm{C}} \approx 2 * 10^{4}$;

Comparison of dispersed phase densities: $\rho_{\mathrm{pL}}=1002 \mathrm{~kg} \mathrm{~m}^{-3} ; \rho_{\mathrm{pH}}=1027 \mathrm{~kg} \mathrm{~m}^{-3}$;
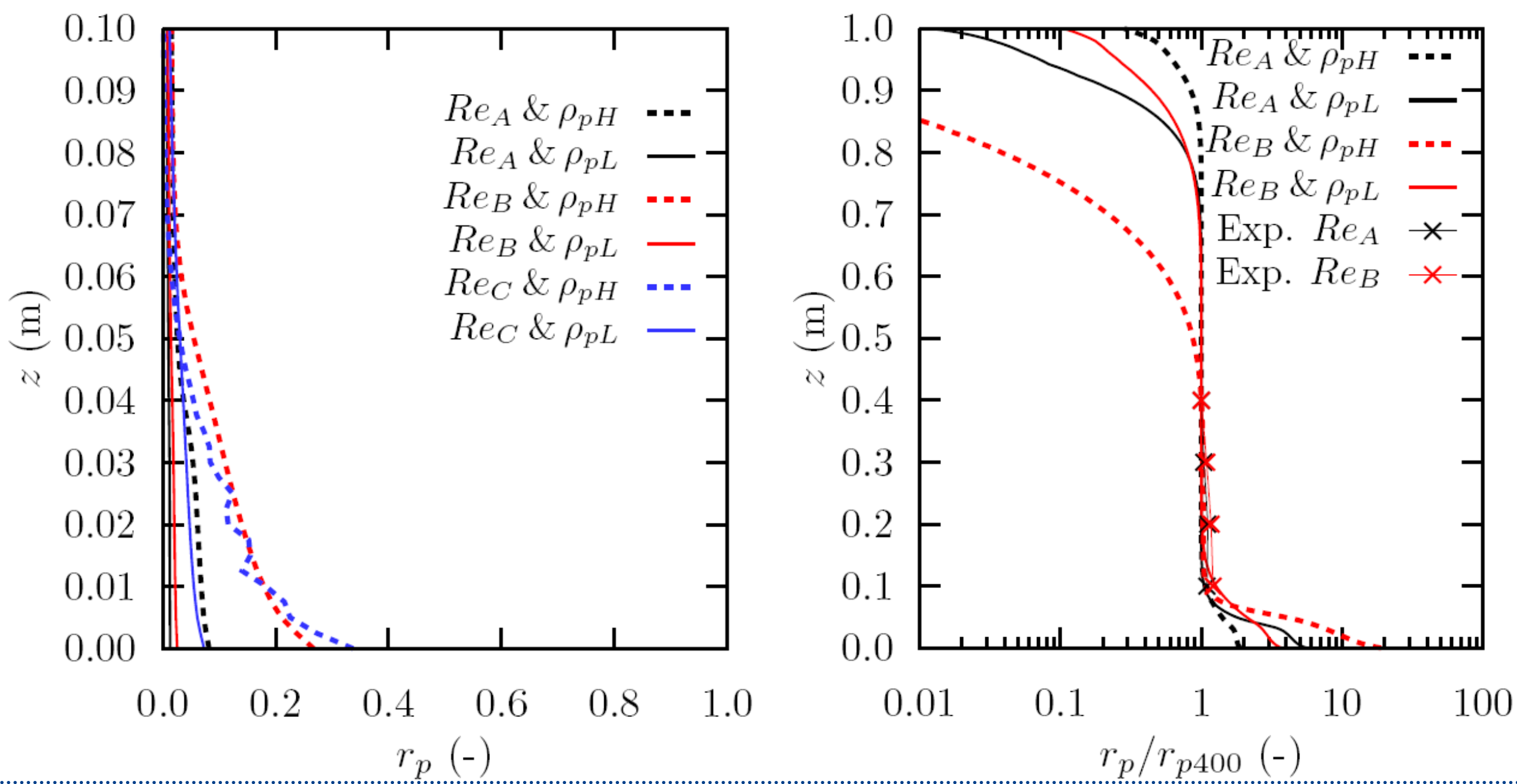


\section{One dispersed phase:}

Interfacial Force Coefficients: $\mathrm{C}_{\mathrm{TD}}=100 ; \mathrm{C}_{\mathrm{D}, \mathrm{SNW}}$;

Three inlet velocities: $\mathrm{Re}_{\mathrm{A}} \approx 10^{5} ; \mathrm{Re}_{\mathrm{B}} \approx 4^{*} 10^{4} ; \mathrm{Re}_{\mathrm{C}} \approx 2^{*} 10^{4}$;

Comparison of dispersed phase densities: $\rho_{\mathrm{pL}}=1002 \mathrm{~kg} \mathrm{~m}^{-3} ; \rho_{\mathrm{pH}}=1027 \mathrm{~kg} \mathrm{~m}^{-3}$;
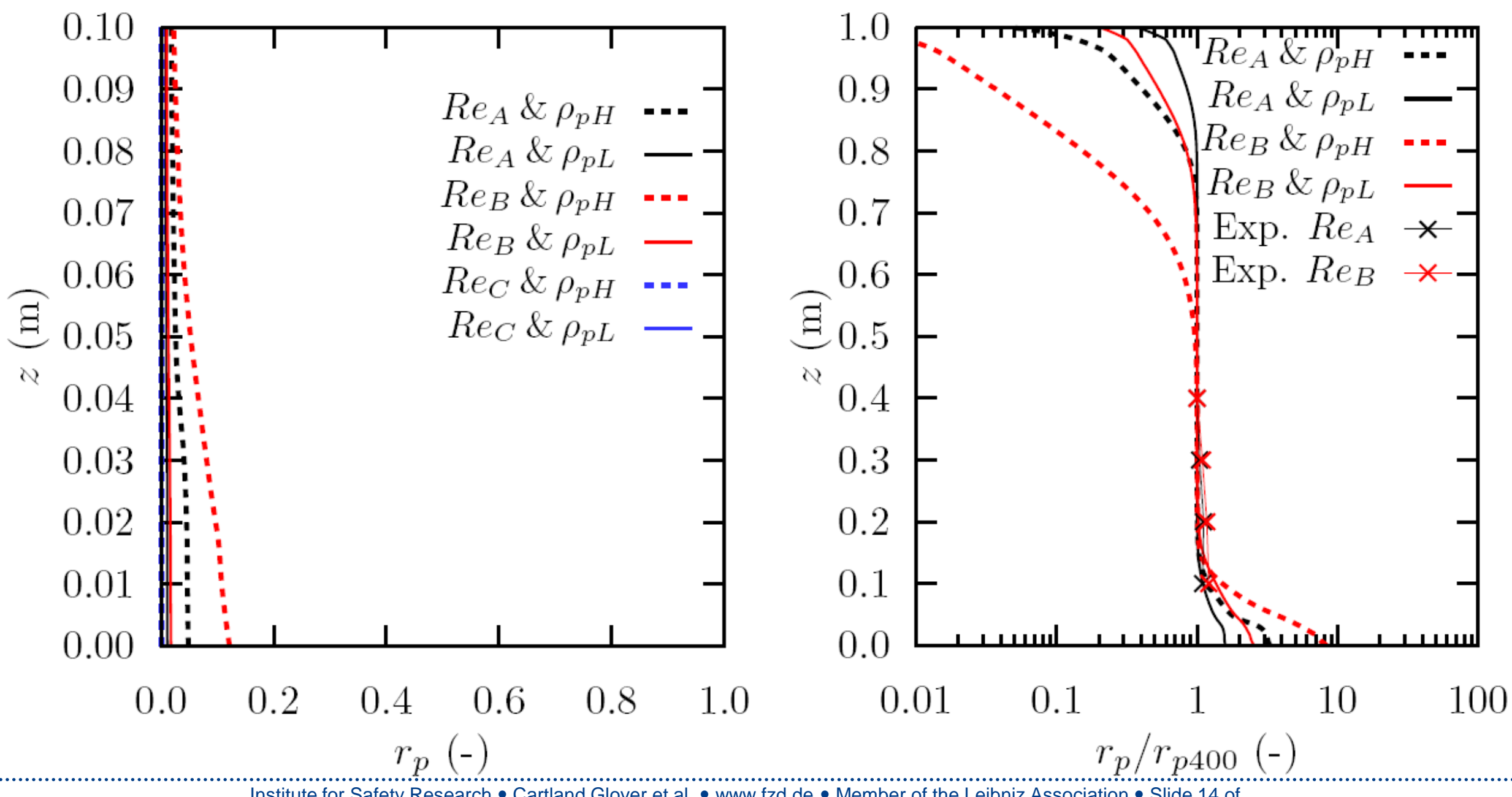


\section{Two dispersed phases:}

Drag Coefficient: $\mathrm{C}_{\mathrm{D}, \mathrm{SN}}$;

One inlet velocity: $\operatorname{Re}_{\mathrm{A}} \approx 10^{5}$;

Dispersed phase densities: $\rho_{\mathrm{pL}}=1002 \mathrm{~kg} \mathrm{~m}^{-3} ; \rho_{\mathrm{pH}}=1027 \mathrm{~kg} \mathrm{~m}^{-3}$;
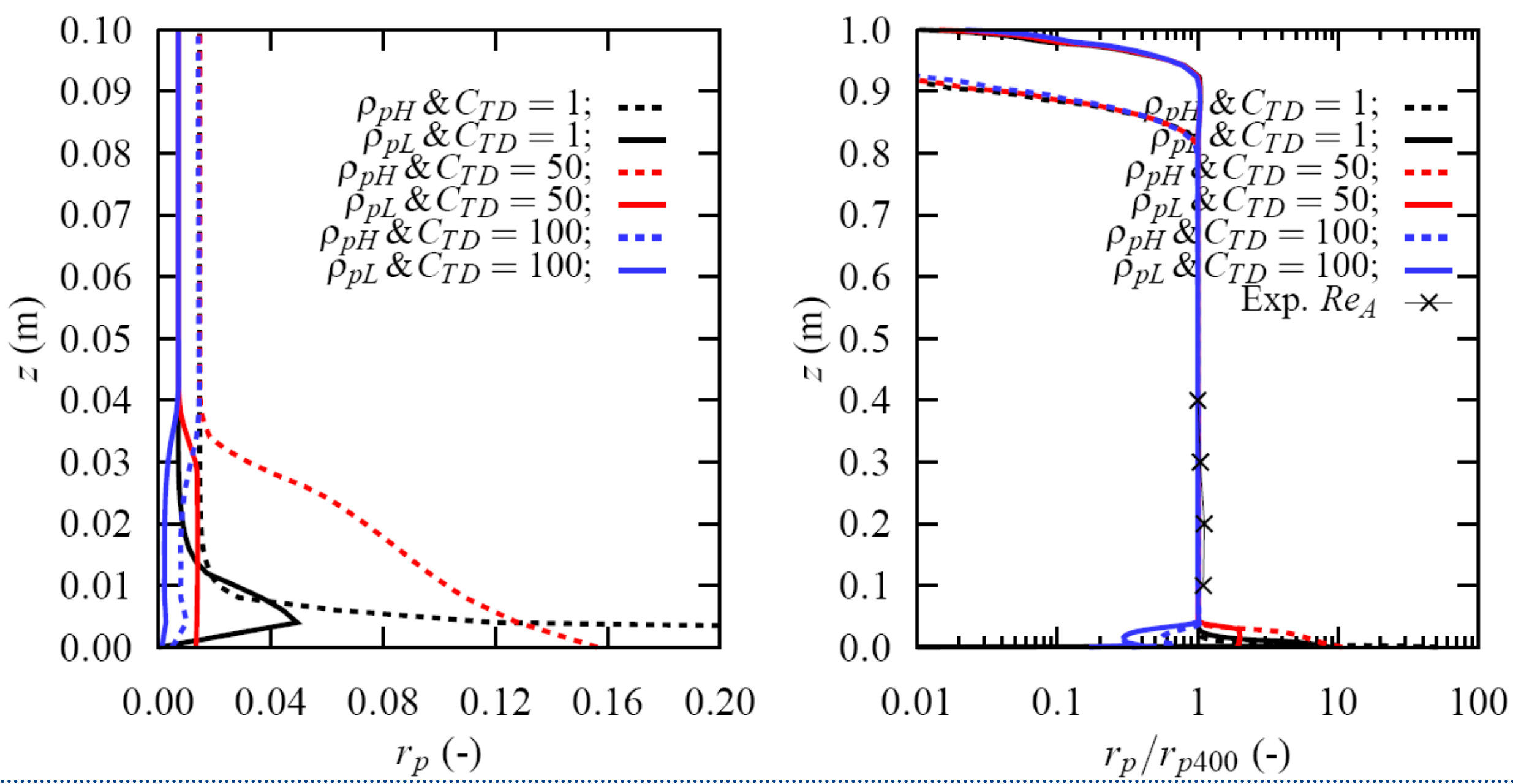


\section{Two dispersed phases:}

Drag Coefficient: $\mathrm{C}_{\mathrm{D}, \mathrm{SNW}}$;

One inlet velocity: $\operatorname{Re}_{\mathrm{A}} \approx 10^{5}$;

Dispersed phase densities: $\rho_{\mathrm{pL}}=1002 \mathrm{~kg} \mathrm{~m}^{-3} ; \rho_{\mathrm{pH}}=1027 \mathrm{~kg} \mathrm{~m}^{-3}$;
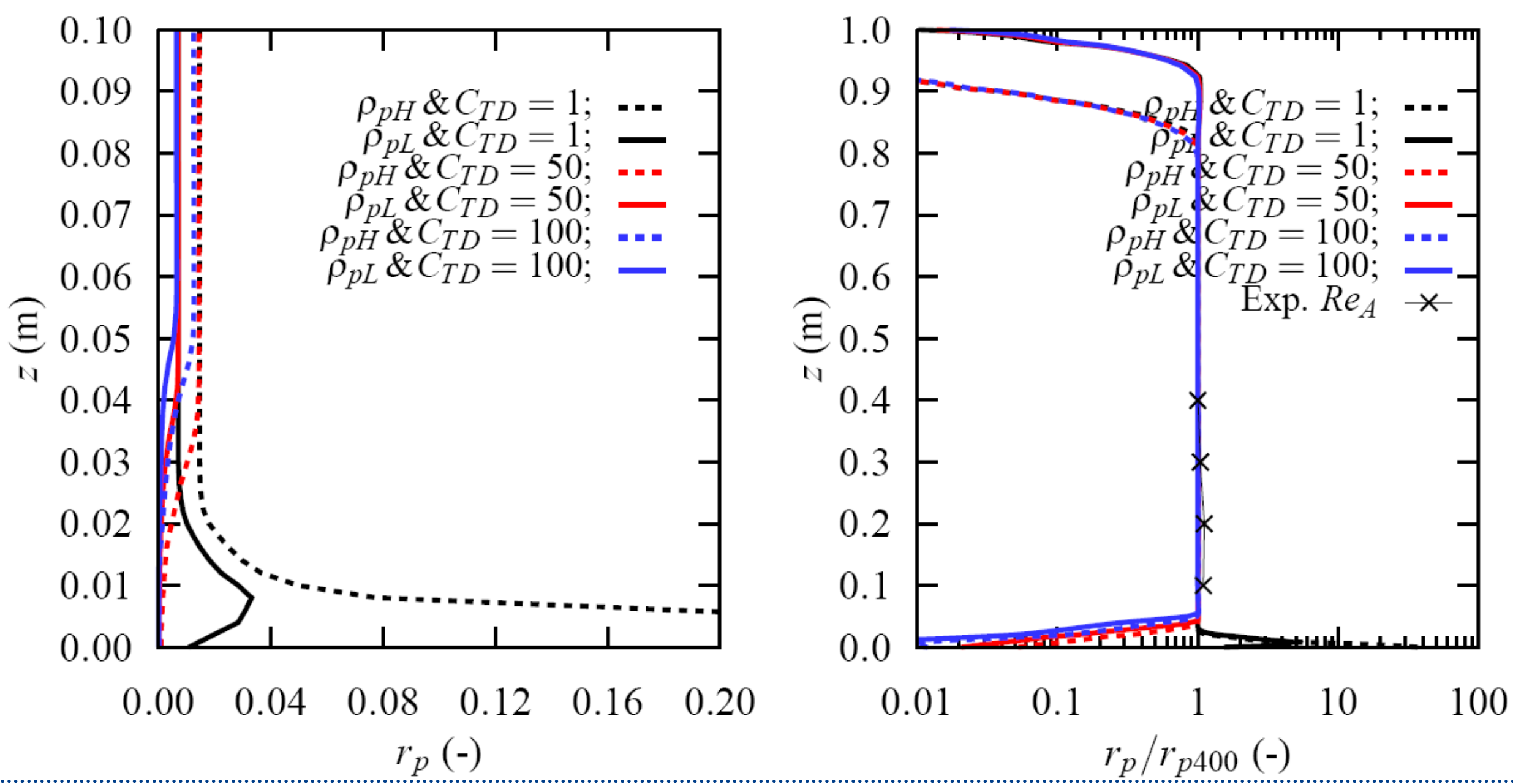
- Simulations mimic fiber transport in a section of a racetrack type channel.

- The method offers a technique to model fiber transport in NPP containment sumps.

- The experimental results show that the numerical concepts can capture fiber transport.

- Fibers accumulate in the lower boundary layer at all velocities

- Fibers do not accumulate at the highest velocities in the experiments

- Modifications applied to interfacial force coefficients cause reductions in fiber accumulation in the lower boundary layer.

- Large $\mathrm{C}_{\mathrm{TD}}$ cause fibers wash out of the channel at lower velocities.

- Continuous phase gradient affects the turbulent dispersion force

- As smaller turbulent dispersion coefficients when two dispersed phases are modeled simultaneously.

- Uniform velocity and volume fraction profiles were employed at the inlet condition.

- Turbulent dispersion force is strongly dependent on local turbulence field. 


\section{Future Work}

- Detailed experiments are required

- More detail in the lower boundary layer

- Establish a quasi steady state at the lower velocity conditions

- Size distributions in the channel

- Better characterization of the relative viscosity

- Thorough validation of the numerical models with further consideration of

- Number of dispersed phases

- Dispersed phase density

- Dispersed phase size

- Dispersed phase fraction

- Use of non-uniform boundary conditions on the channel section

- Model the whole channel 